\title{
Integrated clinical pathway management for medical quality improvement - based on a semiotically inspired systems architecture
}

\author{
Weizi Li ${ }^{1}$, Kecheng Liu ${ }^{1}$, \\ Hongqiao Yang ${ }^{1}$ and \\ Changrui $\mathrm{Yu}^{2}$ \\ ${ }^{1}$ Informatics Research Centre, Henley Business \\ School, University of Reading, Reading, U.K.; \\ ${ }^{2}$ Shanghai University of Finance and Economics, \\ Shanghai, China
}

Correspondence: Weizi Li, Informatics Research Centre, Henley Business School, University of Reading, Whiteknights, Reading, RG6 6UD, U.K.

Tel: +440 118378 4281;

E-mail: weizi.li@henley.ac.uk
Received: 03 June 2011

Revised: 06 October 2011

2nd Revision: 08 February 2012

3rd Revision: 15 August 2012

4th Revision: 28 January 2013

Accepted: 15 April 2013

\begin{abstract}
Clinical pathway is an approach to standardise care processes to support the implementations of clinical guidelines and protocols. It is designed to support the management of treatment processes including clinical and non-clinical activities, resources and also financial aspects. It provides detailed guidance for each stage in the management of a patient with the aim of improving the continuity and coordination of care across different disciplines and sectors. However, in the practical treatment process, the lack of knowledge sharing and information accuracy of paper-based clinical pathways burden health-care staff with a large amount of paper work. This will often result in medical errors, inefficient treatment process and thus poor quality medical services. This paper first presents a theoretical underpinning and a co-design research methodology for integrated pathway management by drawing input from organisational semiotics. An approach to integrated clinical pathway management is then proposed, which aims to embed pathway knowledge into treatment processes and existing hospital information systems. The capability of this approach has been demonstrated through the case study in one of the largest hospitals in China. The outcome reveals that medical quality can be improved significantly by the classified clinical pathway knowledge and seamless integration with hospital information systems.

European Journal of Information Systems (2014) 23(4), 400-417.

doi: 10. I057/ejis.2013.9; published online 28 May 2013
\end{abstract}

Keywords: clinical pathways; integrated systems; co-design; medical quality improvement; organisational semiotics; system architecture

The online version of this article is available Open Access

\section{Introduction}

Clinical pathways - also known as integrated care pathways, coordinated care pathways, care maps or anticipated recovery pathways - are taskorientated care plans that detail essential steps in the care of patients with a specific clinical problem and describe a patient's expected clinical course (Coffey et al, 1992; Kitchiner \& Bundred, 1996). The concept was introduced for the first time at the New England Medical Centre (Boston, U.S.A) in 1985, originally by Karen Zander and Kathleen Bower. Clinical pathways is a result of adapting documents used in industrial quality management known as Standard Operating Procedures that aims to improve efficiency in the use of resources and task completion within a set time. Clinical pathways offer a structured approach in developing and implementing local protocols of care, based on evidence-based clinical guidelines. They also provide a means 
of identifying the reasons why clinical care falls short of adopted standards, the 'missing link' in audit projects (Crombie \& Davies, 1993).

Clinical pathways differ from clinical guidelines and protocols as they are a set of practical treatment processes detailing how to implement clinical guidelines, including both clinical guidelines and non-clinical activities. They are used by a multidisciplinary team and for coordination of care. The clinical pathways are usually designed and monitored by a hospital pathway committee. Physicians use clinical pathways to give appropriate instructions on a daily basis. The nurses review the orders and confirm their accomplishment. Other care team members such as the therapist, nutritionist, interns and clerks carry out all the orders in the pathway pertaining to them. Patients' progress in the pathway will be reviewed and the variances will be recorded by nurses and physicians. The variance will be monitored and analysed by the pathway team (e.g., pathway committee) to discover the areas for improvement. This will help in increasing outcome consistencies. Pathways thus promote continuous improvement and reliable care. In addition, clinical pathways help in pursuing the goals of improving patient care quality, maximising the efficient use of resources and supporting clinical effectiveness of health-care staff.

Although the applications of clinical pathways improve the quality of care, there are still shortcomings in practical patient treatment behaviour. One of the drawbacks is the lack of knowledge sharing and computerisation of pathway information. International surveys reveal that knowledge sharing on how multidisciplinary teams organise care processes becomes a big issue (Vanhaecht, 2007). Moreover, paper-based pathways are challenging for knowledge sharing and burdensome paper work causes inefficiency in care processes. Although the development of hospital information systems has provided opportunities of computerisation and knowledge sharing, the separation of pathway knowledge from various information systems without sharing a common ground is a major problem for knowledge management in health-care organisation (Muscholl, 2005; Blaser et al, 2007; Abidi, 2008). Even though decision support intelligence has been implemented in some of the hospital information technology (IT) applications (Elson \& Connelly, 1995; Lenz \& Kuhn, 2004; Blaser et al, 2007; Friedlin et al, 2007; Lobach et al, 2007; Mathe et al, 2009), they can only support certain parts of medical practice instead of the whole treatment process. Barjis et al (2010) mention that the integration of decision models within business processes is one of the challenges in knowledge management.

The lack of information accuracy in the treatment process is another weakness of clinical pathways. Although clinical pathway could reduce unnecessary documentation to some extent, the long and complicated documents often cause inappropriate data input within the multidisciplinary team. In particular, in circumstances where a patient has complications or more than one disease, the lack of pathway knowledge sharing and burdensome paper work could easily lead to duplicate therapy, inappropriate dose and drug interaction. Those medical errors will have adverse consequences for patients. According to a study conducted in two London teaching hospitals, it has been found that $10 \%$ of patients out of 1014 admissions experienced an adverse event, where half of it is preventable, adding a mean of 8.5 additional days in hospital, incurring additional costs of $£ 367894$ (Vincent et al, 2001). A study of more than 4000 admissions in two U.S. tertiary care centres found that in almost $2 \%$ of cases, there was a preventable adverse drug event, resulting in a mean increased hospital stay of 4.6 days and additional costs of $£ 4746$.

In order to overcome the aforementioned issues, it is necessary to computerise clinical pathways and integrate pathway knowledge with existing information systems such that the shared pathway knowledge can provide seamless support in the treatment. Instead of the isolated and error-prone paper-based pathways, the computerised and shared pathway throughout the treatment process will help in achieving a patient-centric process, improving care coordination and efficiency, as well as reducing medical errors. This paper first presents a theoretical underpinning and the co-design research methodology for an integrated pathway management approach by drawing input from organisational semiotics (OS). The integrated clinical pathway management approach is then proposed, aiming to integrate clinical pathway knowledge into treatment process and existing hospital information systems. The paper is organised as follows: the next section 'Theoretical background and research methodology' describes the complexity of treatment process that leads to the introduction of OS theory underpinning and the co-design methodology; the subsequent section 'The integrated clinical pathway management through co-design process' provides a detailed integrated system design through the co-design methodology; the case study of applying the system for improving medical quality in one of the largest hospital in China is presented in the section after that, 'Evaluation: A case study'; the related work in comparison with this research is discussed in the penultimate section, 'Related research'; the conclusion about the use of an integrated management system and a suggestion for further development are set out in the final section 'Discussion and conclusions'.

\section{Theoretical background and research methodology}

\section{Motivation of co-design methodology - the complexity of treatment processes}

In this section, we will discuss the complexity of clinical processes that will lead to the co-design methodology to support the complex processes. The complexity of the treatment process is manifest in many ways including various types of activities, different levels of knowledge and processes, as well as coordination among stakeholders. The core part of the treatment process is clinical pathways 
where various clinical activities are defined in certain sequences. These activities include clinical evaluation, inspection, medication, laboratory test, surgery and so on, which are formalised in the form of medical documents. These pathways should be patient-specific and dynamic with patient's evolving status. The pathway knowledge is the 'knowing' aspect of the treatment process, but currently this is static in paper-based documents. On the other hand, each activity in the pathway is also supported by several sub-activities. For example, the laboratory test in clinical pathway will be supported by several subactivities such as test request, reporting, result evaluation and record and so on, which are daily routines relying on organisational knowledge and are mostly automated by the current hospital information systems. They are the 'doing' aspect of the treatment process involving coordination among stakeholders and systems but mostly are separated from medical knowledge and patient status. Therefore, the treatment processes are composed of the interactions and coordination between various activities in different levels of activity and multiple types of knowledge. This is what leads to the complexity of clinical pathway management. One major problem resulting from the complexity is the separation of the formal clinical pathway knowledge and the activities supported by the technical information systems in the treatment processes, also called 'knowing-doing gap' or 'clinical pathway/ guideline implement gap' (Muscholl, 2005; Abidi, 2008). Furthermore, current systems in the hospital such as laboratory information system, electronic patient record, radiology information system and so on are also separated and hence cannot support the complex clinical processes seamlessly. Therefore, an approach of designing the process knowledge and the system together is important in order to fill the gap of clinical pathway implementation. In this research, we propose a co-design approach that designs the clinical pathway knowledge and the pathway management system in parallel to integrate the pathway knowledge seamlessly into the treatment process.

\section{Organisational morphology: theoretical background} From the perspective of OS (Stamper, 2001), an organisation is regarded as an information system. The organisation is able to provide services and perform functions that are determined by the organisation's capability (or affordance). Most of these service capabilities and functions are explicitly defined and described. They are regulated or governed by explicit norms (e.g., business rules). These are called formalised activity or formalised behaviour. Within the whole range of formalised organisational behaviours, some services and functions are mechanistic and can be automated by computer-based systems. In such cases, technical solutions or automatic systems can be employed to perform these functions instead of humans. Semiotic approach suggests that the IT system is part of a formal part of the organisation that is, in turn, part of the total organisation (Liu, 2000). In this way, IT is seen as

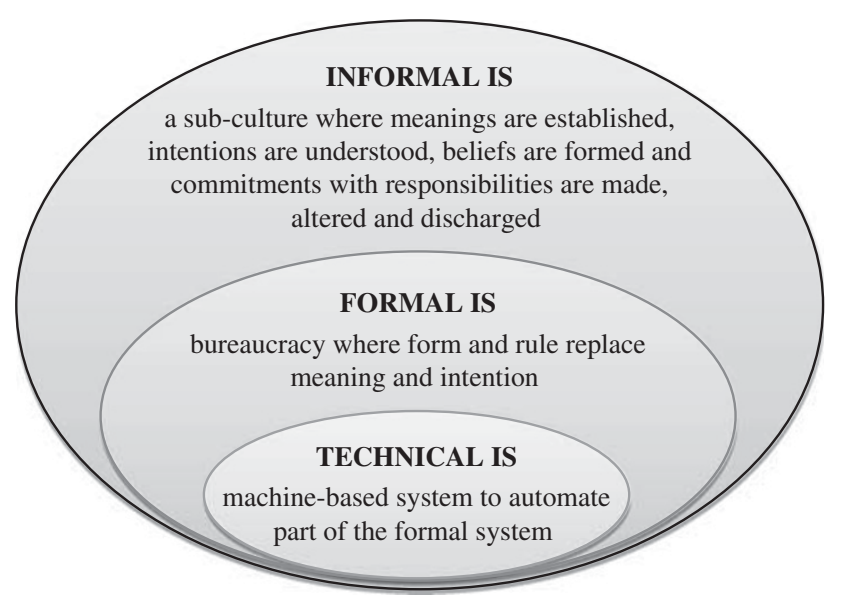

Figure 1 The organisational onion (Liu, 2000).

part of the entire organisation and should be subject to the formal and informal aspects of the business. The organisational morphology can be described as the organisational onion as shown in Figure 1.

The informal level: Organisational culture, customs and values are reflected as beliefs, habits and patterns of behaviour of each individual member. At this level, meanings are agreed, intentions are understood and beliefs are formed. Commitments with responsibilities are made, altered and discharged in this context through negotiation, discussions and physical actions.

The formal level: Rules and procedures are created to replace meanings and intentions. The rules and formal procedures specify how the work should be conducted.

The technical level: Represents the computer application placed inside the formal system layer. The technical system presupposes a formal system, just as a formal system relies on an informal system.

This holistic view of organisations, people, norms and IT allows a comprehensive analysis about how organisations run their business and therefore provides a theoretical architecture for clinical pathway management in healthcare organisations. Hospital strategy and missions, such as medical error reduction and quality improvement, represent the intentions and beliefs they share. There are many aspects of treatment behaviours that influence the hospital as a whole, derived from informal practices shared by the health-care staff. It is emphasised that the implementation of clinical pathway comprises more than just the structure of the care process. Pathways in practice involve complex activities that are the combination of individual interventions in the informal level suited to organisation needs, ability and culture (Vanhaecht, 2007). In particular, patient safety culture in a hospital is an important informal factor that centres on the actual provision of care and process that impact care quality and safety (Stronks \& Galvez, 2007). An effective patient safety culture refers to patterns of individual and organisational behaviour, based 
on shared beliefs and values, that continuously seeks to minimise patient harm that may result from the process of care delivery (Kizer, 1999). Apart from the informal activities in the care processes, it is more crucial that the pathway knowledge is explained to and shared by the multidisciplinary team. The pathway knowledge represents the formal aspect of the onion. These processes and procedures guide, direct and control health-care staff behaviour in an explicit way, hence they are able to consolidate the knowledge and bureaucracies in the hospital. The technical aspect is implemented by the IT applications such as the physician's work station, radiology information systems and so on. Some formal treatment activities are so well formalised that they can be automated by machines.

On the basis of the organisational onion, informal, formal and technical levels must be combined together for seamless support. Therefore, the practical treatment process and the cohesive patient safety culture, clinical pathway knowledge and existing health-care IT systems need to be integrated to provide a comprehensive clinical pathway management in order to improve medical quality. Organisational morphology is applied as the theoretical foundation in this research. Furthermore, based on this theoretical perspective, this research adopts the co-design methodology as presented in the section 'Co-design methodology based on OS'.

\section{Co-design methodology based on OS}

The concept of co-design was first proposed by Liu et al (2002) to minimise the gap between business and IT systems. This concept is based on OS that considers an IT system to be designed as part of the organisation. From the co-design principle, IT systems must meet the business requirements, support the business process and adapt to changes in business practices. Therefore, based on the organisational morphology and co-design concept, we have adapted the co-design methodology for designing the clinical pathway management system as a part of the treatment process. The clinical pathway knowledge and the integrated management system are designed in parallel. The co-design methodology is described in Figure 2.

As shown in Figure 2, the co-design methodology is composed of three phases: (1) problem scoping phase in informal level, (2) co-design of formal pathway and technical system for the integrated system (3) evaluation.

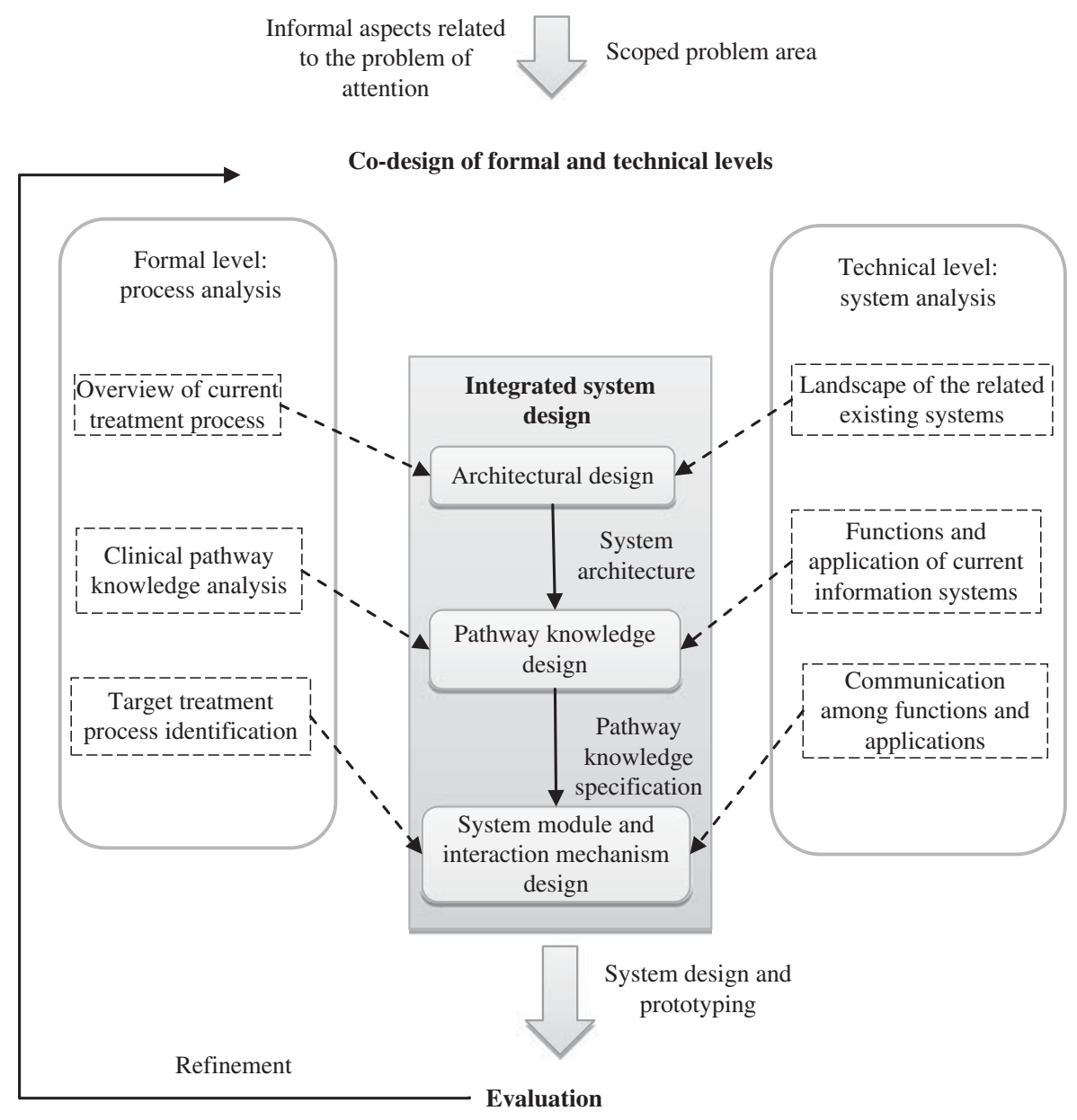

Figure 2 Co-design methodology in clinical pathway management. 
These three phases constitute the design processes where the gaps between informal, formal and technical levels will be minimised and the integrated system combining pathway knowledge and existing information systems will be designed.

The problem scoping phase focuses on an understanding of the informal level of the organisation that identifies the business requirements and the problem that needs to be solved. This problem scoping phase defines the problem space where organisational practice, stakeholders, culture, patterns of behaviour, strategy and opportunity reside in. Together these define the business needs or problem. In this phase, business problems are analysed and scoped within the context of organisational strategies and practice, culture, stakeholders and patterns of behaviour. The outcome of this phase leads to a prioritised scope of the business issues to be addressed in the co-design. In this research, we first identify the issues on the lack of knowledge sharing and information accuracy in clinical pathway management that is discussed in the section 'Introduction'. This guides the following co-design process for the integrated clinical pathway management. Furthermore, there are different informal factors such as leadership, workforce, language, community engagement, quality improvement and patient safety. They influence the provision of care through business strategy, culture, stakeholders' beliefs and patterns of behaviour. As discussed in the section 'Organisational morphology: Theoretical background', we identify the patient safety and quality improvement culture as the most related informal factors in clinical pathway management. At the same time, methods to promote patient safety culture to improve medical quality also need to be identified as the informal part of the integrated system. More existing research on patient safety culture to improve medical quality is found in section 'Discussion and conclusions'.

The co-design phase focuses on the integrated system design tightly coupled with the formal pathway knowledge and technical systems, which aims to align the treatment processes and the system design seamlessly. The co-design phase includes three stages: architectural design, process design, and the design of system module and interaction mechanism.

- The architectural design takes a structural view of the current treatment process and the landscape of the existing information systems in the problem scope. The section 'Motivation of co-design methodology the complexity of treatment processes' presents a brief discussion on the overview of treatment process and current systems. By considering the overview from both the formal and technical aspects, the integrated system architecture will be designed combining both aspects as the output of this stage. This is a high-level architecture that leads to an overall picture about how the integrated system supports different levels of treatment process.

- The pathway knowledge design specifies the formal pathway in the form that can be fully supported by the proposed integrated system. In this stage, related formal pathway knowledge will be identified and analysed. In the meantime, the inventory of functions and applications of the existing technical systems, as well as the corresponding services and activities supported by the applications, will also be identified. As a result of considering the two aspects concurrently, the pathways knowledge from related sources in different modalities, for example, tacit knowledge, explicit knowledge, experience, recorded discussion and observations, protocols, educational resources and procedures, will be extracted, modelled and specified in forms such as workflow, activities, norms, rules and constraints that can be understood by the integrated system.

- The system module and interaction mechanism design will lead to the design of the system module and their interactions. In this stage, the target treatment processes supported by the integrated system and the way of communication among the existing technical systems are identified and analysed. Furthermore, based on the specified pathway knowledge in the previous stage and the combined view of the formal process and technical communication, the modules and their interaction mechanism for coordinating different stakeholders and system applications will be designed. In particular, the interoperability and communication standards within the integrated system and current applications will be considered in this stage.

The evaluation phase will focus on evaluation of the efficacy of the integrated system. The evaluation and the measurement is conducted from different dimensions of the application domain, leading to further refinement of the co-design process. In this research, the case study method is used to evaluate our approach from technical and medical quality dimensions. The technical dimension of the approach is demonstrated by the trial implementation of the system in clinical pathways. The impact on medical quality, as well as the cost and efficiency of treatment process, is also evaluated through the case study. Co-design methodology is an iterative process therefore the evaluation result will be used to refine the integrated system from problem scoping to system modules design.

In this paper, we focus on the integrated system design in the co-design phase and the case study in the evaluation phase. The detailed design in each stage will be discussed in the section 'The integrated clinical pathway management through co-design process' and the case study of the integrated clinical pathway management system in a hospital will be presented in the section 'Evaluation: a case study'.

\section{The integrated clinical pathway management through co-design process}

\section{Architectural design}

As shown in Figure 3, the system architecture for the integrated clinical pathway management is proposed 


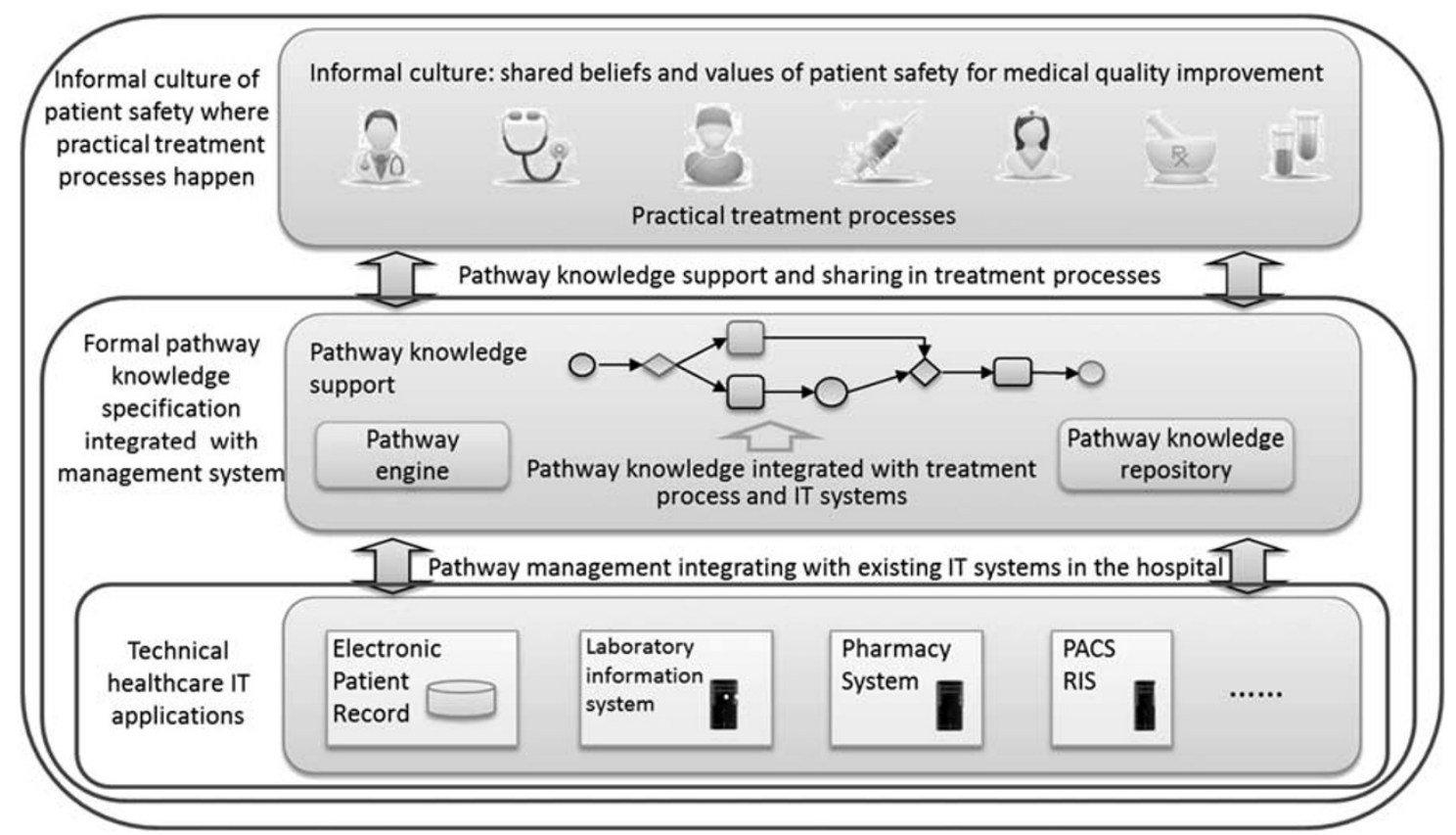

Figure 3 System architecture for the integrated clinical pathway management.

based on OS that regards information systems as a part of the organisation and the co-design process. In this architecture, the informal level where practical treatment process happens, specified formal pathway knowledge and the technical healthcare IT systems are integrated in order to provide seamless pathway knowledge support at every step of treatment process, as well as to coordinate the multidisciplinary team in the care process.

In order to improve medical quality through the integrated system, the informal level where practical treatment process happens will be consolidated by promoting patient safety culture. Therefore, in this level, the shared beliefs and values to treat patients safely and successfully will be established. This further enables the cohesive culture of high medical quality and impacts on health professionals' behaviour throughout treatment processes. New medical quality improvement strategy and the updated clinical pathway implementation guidelines can be seen as the informal actions to improve medical quality. The changing strategy and guidelines could help to promote the culture of reducing medical errors. For example, staff in all departments in the hospital can be encouraged to improve their prescribing and recording accuracy by being more specific and detailed.

The formal level in the middle with the specified pathway knowledge plays a central role, both supporting the informal treatment process and connecting with the current information systems. In this level, pathway knowledge is specified in the knowledge repository and the pathway engine supports the treatment process based on the predefined knowledge and patient information. Together they provide health-care staff with pathway information in the informal treatment process level and support the execution of each step as well as cooperation within the treatment team. On the other hand, the formal pathway knowledge is also integrated with the existing health-care IT systems in the technical level. The pathway engine communicates with various information systems according to different activities during the execution of pathway. Furthermore,, by integrating with the current systems, the pathway engine is able to give appropriate pathway information based on real-time treatment information for health-care staff in the informal treatment level. In this paper, we will focus on the co-design of formal clinical pathway knowledge and the integrated pathway management system. The informal level on how to build an effective culture will be discussed in the section 'Discussion and conclusion'.

\section{Pathway knowledge design}

The clinical pathway knowledge is elicited and specified by analysing multiple sources of where we consider: (1) tacit knowledge of domain experts; (2) published literature on clinical pathways; (3) existing clinical pathways in use currently in hospitals; and (4) clinical data in current hospital IT applications. The current research on clinical pathway modelling mainly focuses on the ontological structure (Hurley \& Abidi, 2007). Baacke et al (2009) develop the component-based process modelling method with an emphasis on the differentiation of generic activities (the daily routine information processing activities) and processed objects (the medical objects), but there has been very little work done on detailing classification and specification of clinical pathway knowledge. A modelling method for clinical pathways and the customisation 
mechanism with an emphasis on analysing and classification process is developed that will lead to a more comprehensive medical knowledge base (Li et al, 2008). The clinical pathway knowledge is classified along two axes: clinical facts and clinical rules. The classification of clinical pathway knowledge along these two axes ensures comprehensive support for the execution of clinical pathways.

The clinical pathway knowledge representation design captures the health-care professional's knowledge such as the decision and experience knowledge for certain pathways in the form of facts and rules. The facts describe all activity types in a certain pathway associated with the responsible personnel and departments. In this research, the treatment facts are classified into 10 main types according to the long-term treatment experience and analysis of a large number of clinical records. These 10 types of treatment facts cover all activities involved in clinical pathways. These activities are: (1) medication: the recommended medicine for certain diseases; (2) clinical evaluation: documentation for the evaluation of a patient's condition, examination and test results; (3) nutrition: the recommended meals for a patient with a certain disease; (4) observations: documentation for a patient's vital signs such as blood pressure and temperature; (5) activity: the behaviour constrains for a patient with a certain disease such as absolute bed rest; (6) nursing: different levels of nursing and education; (7) surgery: surgery types for certain diseases; (8) examination: the recommended examinations for certain diseases; (9) consultation: consultation content for certain diseases; (10) variation: the record of a patient's condition deviating from the standard pathway record.

The clinical rules on the other hand specify the details of these activities, such as when and how certain clinical activities should happen. On the basis of research on a substantial number of clinical pathways, rules are classified into pathway rules and activity rules. Pathway rules control the process in clinical pathways, which consist of the following (1) single disease treatment indices rules: the quality criteria of certain disease issued by the national health department that needs to be achieved through treatment; (2) pathway application criteria rules: the rules determining whether a patient is suitable for a certain pathway; (3) activity steps sequences rules: the logical sequential and parallel relation among the steps of clinical pathways; (4) activity step rules: rules suggesting the medical activities in each step in the form of a set of if-then rules; (5) step reminder rules: triggering an intelligent reminder when a certain step is going to be executed; (6) pathway selection rules: rules determining which branch of the pathway to execute. The activity rules refer to the detailed application conditions and constraints of classified medical activities such as medication, examination and other activities in facts bases. As most of the medical activities have their own indications and contradictions, this information could be fed into decision support, hence effectively reducing human error and
Table 1 Example of typical knowledge bases

\begin{tabular}{|c|c|}
\hline Knowledge bases category & $\begin{array}{l}\text { Number of } \\
\text { items }\end{array}$ \\
\hline Medication services & 16350 \\
\hline Drug-drug interactions for $\mathrm{CPOE}$ & 2000 \\
\hline $\begin{array}{l}\text { First databank Drug-drug interactions/allergy tables } \\
\text { for pharmacy and CPOE }\end{array}$ & 4000 \\
\hline Geriatric expert dosing & 100 \\
\hline Renal expert dosing & 250 \\
\hline Paediatric and neonatal expert dosing & 2000 \\
\hline $\begin{array}{l}\text { Drug dictionary with venue/clinical discipline specific } \\
\text { mappings }\end{array}$ & 8000 \\
\hline Other order-entry related decision support & 1230 \\
\hline Consequent lab display & 60 \\
\hline Consequent orders & 60 \\
\hline Indication screened orders & 20 \\
\hline Antibiotic restriction/decision support & 40 \\
\hline Drug-lab prescribing error decision support & 350 \\
\hline Drug-problem prescribing error decision support & 700 \\
\hline Ambulatory problem list database & 1000 \\
\hline Physician order entry knowledge bases & 2100 \\
\hline In-patient order sets/templates & 600 \\
\hline $\begin{array}{l}\text { Chemotherapy protocols (investigational and } \\
\text { therapeutic) }\end{array}$ & 1500 \\
\hline Surveillance and notification alerts & 230 \\
\hline $\begin{array}{l}\text { Pharmacy ADE and potential Adverse drug event } \\
\text { (ADE) notification alerts }\end{array}$ & 80 \\
\hline $\begin{array}{l}\text { Physician ADE/potential ADE notification alerts - } \\
\text { in-patient }\end{array}$ & 20 \\
\hline $\begin{array}{l}\text { Physician result notification handling alerts - } \\
\text { outpatient }\end{array}$ & 100 \\
\hline Health maintenance and preventive reminders & 30 \\
\hline Physician order entry knowledge bases & $200+$ \\
\hline Ambulatory order templates & $200+$ \\
\hline Specialty and disease-based filters/dashboards & 100 \\
\hline Specialty views of data & $50+$ \\
\hline Clinician population-management dashboards & $50+$ \\
\hline Clinical documentation templates & 1000 \\
\hline Inpatient documentation & $500+$ \\
\hline Outpatient documentation & $500+$ \\
\hline
\end{tabular}

improving treatment quality. Part of the classification of activity rules listed in knowledge bases is shown in Table 1. Taking medical services that have a total number of 16,350 knowledge items, for example, six types of rules, are derived. These rules cover all the medication details such as drug-drug interaction, allergy tables and other drug usage information that provide intelligent decision support for a doctor's prescription. The detailed knowledge classifications cover most of the clinical activities in a clinical pathway so that a comprehensive intelligent reminder can be provided during treatment process. It is 
not only the logical and time sequence of medical activities in clinical pathway that could be managed, but also a set of well classified knowledge bases could be established for the clinical pathway. Therefore, these sets of knowledge bases can support decisions for the whole treatment process and improve medical quality more effectively.

\section{System modules and interaction mechanism design}

The system modules as well as the interaction steps are shown in Figure 4. They are designed to implement the system architecture for the integrated clinical pathway management discussed in the section 'Architectural design'. This system modules design offers a flexible implementation for integrated clinical pathway management. It supports treatment process execution and automation by using the specified clinical pathway knowledge discussed in section 'Pathway knowledge design'. The clinical pathway knowledge is separated from the current hospital IT systems codes. Hence, the pathway knowledge will be easily adopted in the treatment process by hospital stakeholders with a high degree of flexibility and reusability. The system modules are divided into three layers: the interface layer that supports treatment process, the pathway management layer and the data source layer.
User interface layer The upper layer, the user interface is designed to support interaction with the health-care staff in various treatment activities of the practical care process. This allows health-care staff to access the clinical pathway knowledge and provision of pathway execution guidance in the practical process. In real scenarios, most physicians prefer to keep the existing graphical user interface of their workstation or order entry system instead of having to deal with a new one. Therefore, the interface of the integrated clinical pathway management is embedded in the existing hospital systems, such as physician workstation, to ensure seamless integration with treatment process and current information systems.

Data source layer Existing hospital IT applications constitute the data source layer. Many treatment activities such as medication, laboratory tests and surgical operations are being supported by the existing IT systems. They are the data source of patient treatment activities and are from heterogonous information systems. In order to overcome the heterogeneities, the ServiceOriented Architecture is adopted in the data source layer, which componentises functions of existing information systems as services using unified protocol security guarantee and loose coupling interface. Furthermore,

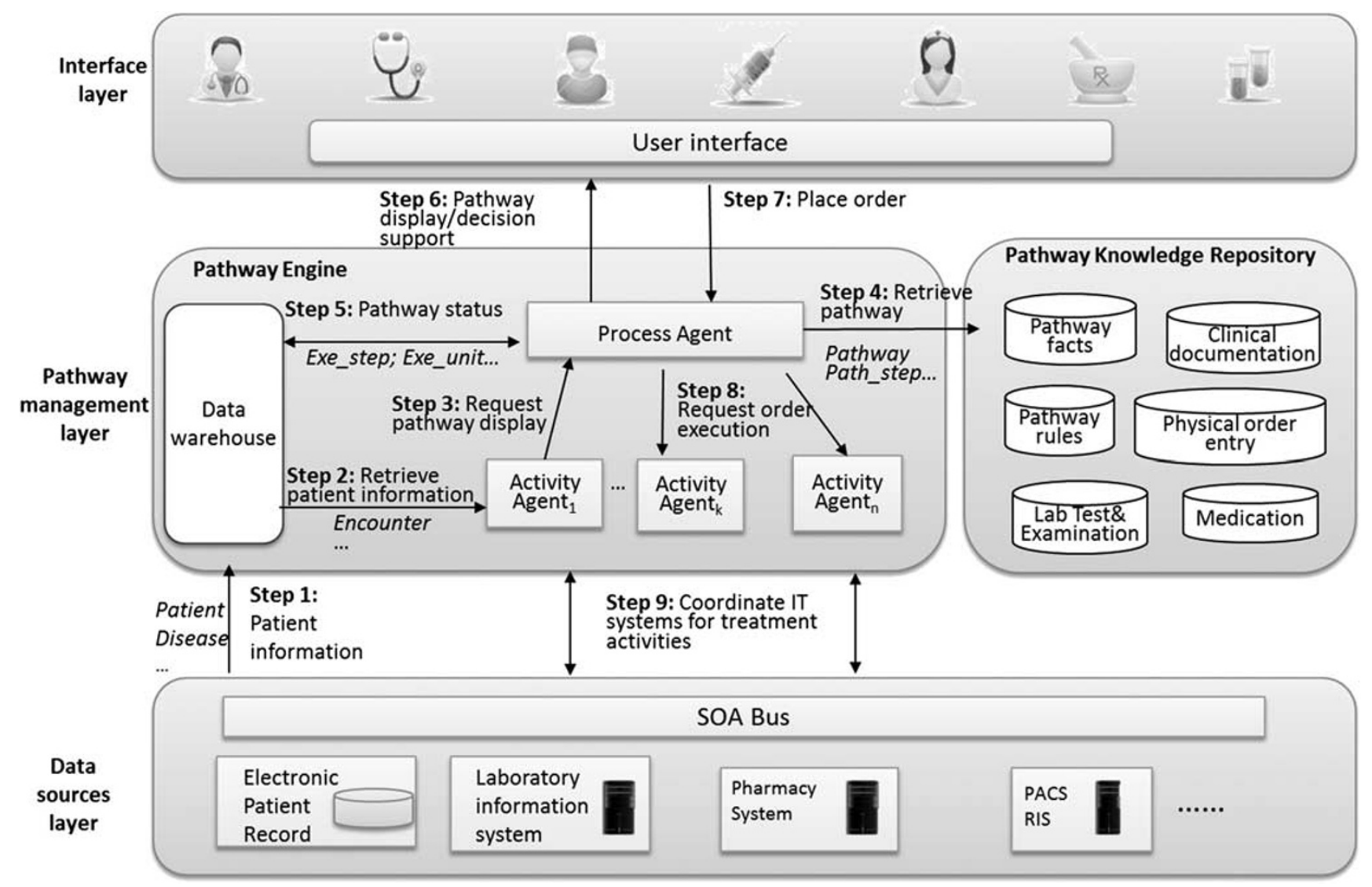

Figure 4 System modules and interactions mechanism for integrated clinical pathway management. 
the middleware of web services are constructed according to the health-care interoperability standards such as Health Level Seven (HL7), DICOM and integrating the health-care enterprise (IHE) in order to achieve information interoperability. Therefore, the complex hospital IT applications and functions can be packaged and integrated seamlessly based on this architecture without reconstructing the existing information systems, in such a way as to preserve the original hospital investment and support flexible medical quality management. This architecture is based on our previous work of the integrated and interoperable health-care systems (Yang \& Li, 2009a; Yang et al, 2010).

Pathway management layer This layer includes the pathway knowledge repository where all the specified pathway knowledge discussed in section 3.2.1 is stored and the pathway engine that implements the knowledge in order to guide and coordinate the execution of the treatment process. Agent technology is adopted in designing the pathway engine. Agent technology has been approved as a good option to be used in developing health-care applications (Isern et al, 2010). In comparison to classical software paradigms, agents offer a natural way of tackling inherently distributed problems with heterogeneous sources, by cooperating and coordinating their activities, and also acting pro-actively to perform tasks that may be beneficial for the user (Nealon $\&$ Moreno, 2003). The main goal of using agents in a pathway engine is twofold. One is to provide the appropriate pathway information to doctors according to patient situation and the treatment progress. The other one is to coordinate the distributed activities between all the health-care staff involved in the treatment process and to interact among the heterogeneous existing hospital information systems.

There are two types of agents: process agent and the activity agents interacting with the existing information systems in data source layer. The process agent enacts the clinical pathway based on the pathway knowledge in the repository and recommends the appropriate treatment activities according to a patient's health status. The activity agents collaborate with the process agent and existing information systems to execute certain activities in the pathway by collecting data and exchanging information. The agents act semi-autonomously. Actions, such as the exchange of information with other systems, collection of data concerning a patient (e.g., past history, test results, treatment activity request and status) are performed by the activity agent without supervision. Actions that may have some degree of responsibility, such as decisions on treatment activities in the pathway, are recommended by the process agent but supervised by the doctors. The agent model structure is based on our previous work on the multi-agent modelling method for clinical pathway management (Li et al, 2009, 2010). All agent functions are implemented over communication protocols and sharing a common pathway knowledge representation specified in

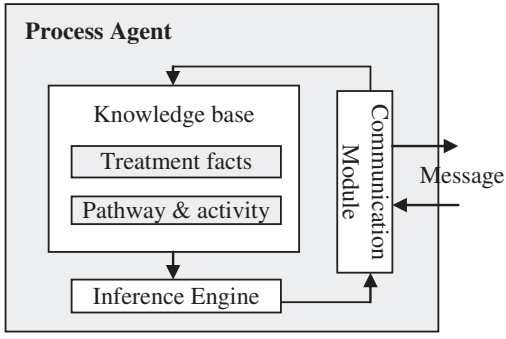

Figure 5 The internal architecture of the process agent.

the pathway knowledge repository. Mainly, agents use two well-known protocols: FIPA-Request and FIPA-ContractNet, and also have an internal knowledge base to represent all exchanged data (FIPA, 2002).

Process agent: The responsibilities of the process agent include: (1) receiving the patient information from the activity agent, (2) searching for the clinical pathway knowledge from the knowledge repository, (3) displaying appropriate treatment process information and knowledge to end users in the graphic interface, (4) invoking the activity agent to interact with the health-care information systems.

The internal architecture of the process agent is shown in Figure 5, which is based on work on the agent-based approach for customised clinical pathway (Li et al, 2010). The communication module receives patient and disease information from activity agents. The knowledge base will then search for the related knowledge including treatment facts, pathway and activity rules from the pathway knowledge repository. The inference engine will produce appropriate pathway information based on the retrieved knowledge and present it to the end user through the communication module. The communication module will further invoke the activity agent to interact with information systems for different medical activities according to the physician's decisions.

There are two types of inference patterns for the inference engine, they are: forward chaining and backward chaining. Forward and backward chaining are reciprocal strategies for reasoning about one part of a decision on the basis of what is known or conjectured about other parts of the decision (Darden, 2002). Forward chaining uses the early stages of a decision to determine the types of entities and activities that are likely to be found downstream. It is driven by data and executed by one or more rules. When there are more than one matched rules, the agenda will execute the predefined activities until all the actions are finished. However, backward chaining reasons from the entities and activities in after stages in a decision to find entities and activities appearing earlier, which is driven by a hypothetical goal, and try to find proper results. If no matched rules can be found, then another sub-goal will be set to look for proper rules until all the results are found or no sub-goals can be found.

The process of clinical decision can be illustrated by forward chaining and backward chaining. The information 
of patient state and treatment progress in the clinical pathway can trigger related rules and provide intelligent decision reminder by forward chaining. However, the execution of clinical pathway needs to achieve single disease quality indices, such as disappearance of certain symptoms, length of stay and treatment cost that can only be achieved by backward chaining. Therefore, the inference mechanism is adopted by combining both forward chaining and backward chaining in the medical knowledge base for making decisions in the treatment process.

Owing to the complexity of matching algorithm of the knowledge bases, the main steps are listed as follows: (1) to input the initial data and goals into working memory, (2) to compare the initial data and goals with the rules via matcher and parser engine, (3) if more than one rule is triggered, then put these rules into a conflict set, (4) to put the triggered rules into the agenda to solve the confliction, (5) to execute the rules in the agenda.

Activity agent: The activity agents play a role of data exchanging and interaction with existing information systems. They act as a bridge between the existing information systems and the process agent. Their responsibilities include: (1) receiving patient information from existing information systems, (2) receiving treatment activity requests from the process agent, (3) invoking information systems to perform treatment activities, (4) updating the treatment status and invoking the process agent to display current progress of the treatment process. The internal architecture of the activity agent is shown in Figure 6.

To integrate clinical pathway into the IT supported treatment process, the interoperability based on relevant standards has been deployed in the activity agent that enables seamless communication among existing information systems. HL7 (2007) develops conceptual standards, document standards, application standards and messaging standards. Such standards set the semantic, structure and data types required for seamless integration from one system to another. IHE Integration Profiles (Vegoda, 2002) describe a clinical information need or workflow scenario and document how to use established standards (e.g., HL7) to accomplish the task so that a group of systems that implement the same Integration Profile address the need/scenario in a mutually compatible way. In this case, the HL7 adaptor in the activity agent works as interface points and exhibits active behaviour as message

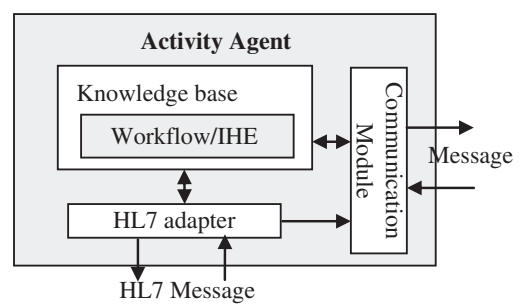

Figure 6 The internal architecture of the activity agent. dispatcher/acceptors handling HL7 messages with hospital information systems. The knowledge base provides information on IHE workflow scenarios and how information systems interact. Whenever the physician decides on any treatment activities through the process agent, the activity agent will interact with the hospital information systems to execute the activity based on the workflow scenario and send an HL7 message through HL7 adapter.

Data storage: The intermediate data extracted from data sources layer and pathway knowledge repository is stored in the data warehouse. The integration framework based on data warehouse for interoperability among heterogeneous health-care systems has been presented in the previous research (Yang \& Li, 2009b).

Three types of data can be combined for communication among agents in supporting the integrated clinical pathway management. They are, data collected from existing hospital information systems, knowledge repository and pathway execution process. The database tables of patient, disease, medicine and inspection item are imported from the information system describing the information on patient, disease, available medicine and inspection in hospital. Pathway, Pathway_Step, Pathway_Unit and Pathway_Item are imported from the knowledge repository describing the detailed clinical pathway information. Encounter, Exe_Step, Exe_Unit and Exe_Item are collected during the pathway execution process describing the status of patient and clinical pathway. The Primary Keys in each table combines these three types of data for agents to integrate the clinical pathway into the treatment process.

Interaction mechanism: Figure 4 also shows the interaction mechanism of the agents in guiding the execution of clinical pathway by integrating the pathway knowledge into treatment process and existing systems.

Step 1: Hospital information systems (e.g., physician/ nurse work station) send the new patient data into Patient and Disease table of data warehouse.

Step 2: The activity agent retrieves and collects patient information from data warehouse as a pathway case into Encounter table.

Step 3: The activity agent invokes the process agent to perform pathway knowledge retrieving.

Step 4: The process agent retrieves the corresponding pathway information, including all the clinical facts and rules from the knowledge repository.

Step 5: The pathway management agent sends the corresponding pathway information to tables of Pathway, Pathway_Step, Pathway_Unit and Pathway_ Item in the data warehouse. The pathway management agent will update the clinical pathway status through Exe_Step, Exe_Unit and Exe_Item table in data warehouse.

Step 6: The pathway management agent displays the clinical pathway information to the physician to guide the execution of treatment process. 
As described above, Steps 1-6 are to ensure decision support of the clinical pathway information based on a patient's situation during the treatment process. Step 7-9 will further integrate the pathway information with existing IT systems to execute treatment activities.

Step 7: Physician selects or enters medical orders (e.g., test, inspection etc.) through user interface based on the recommended pathway information.

Step 8: Process agent invokes activity agents to send message to existing systems in order to execute the treatment orders.

Step 9: The activity agents send messages to corresponding information systems to execute medical orders in the treatment process.

\section{Evaluation: a case study}

\section{Hospital background}

The Hospital of General Staff Department (HGSD) of the People's Liberation Army is one of the largest hospitals in China, with 30 departments for different specialities and having more than 1700 beds. Currently, there are more than 50 subsystems in HGSD that can be classified into nine categories. These categories are physician workstation, nurse workstation, clinical information, anaesthesia management, pharmacy management, office automation, decision support, audio-visual centre and electronic medical record. Physician and nurse workstations, in particular, are two main systems used in the treatment process. All of these systems support different aspects of daily activities in the hospital but most of them are separated.

In recent years, in order to improve medical service quality with effective resource usage at lower risk and cost, HGSD implements clinical pathways in the treatment of six diseases: Non-ST-segment elevation acute coronary syndrome (NSTACS), acute coronary syndrome (ACS), breast cancer, fracture of femoral shaft, ST-segment elevation acute myocardial infarction and uterine leiomyoma. Initially, the clinical pathway knowledge is paper-based, serving a reference purpose without integration with the current information systems. As a result, the management of clinical pathway has become complex and difficult to fully integrate into the treatment process. Medical error, adverse events, unnecessary examinations and tests occur that could be preventable through the support of clinical pathway during the treatment process. Furthermore, because of the isolation of clinical pathway knowledge from existing information systems, it is hard to improve medical quality through the statistical analysis of pathway deviation.

Therefore, it is important that the clinical pathway knowledge is fully integrated into current information systems and is fully transparent to the health-care staff to support the treatment process in improving medical quality. Currently, there are two main problems in clinical pathway management in HGSD. The problems are (1) lack of shared pathway knowledge support causes difficulties in reducing medical errors; (2) lack of integration of IT systems with the formal clinical pathway knowledge causes low efficiency and is error prone. In order to resolve these problems, the integrated clinical pathway management system was implemented in May 2010. This is further illustrated in the subsections below.

\section{Implementation of the integrated clinical pathway system}

The integrated clinical pathway management has been implemented in HGSD. On the one hand, a new strategy for medical quality improvement has been issued. Staff meetings are organised to enforce the safety culture and efficient treatment process from the informal aspect. On the other hand, the clinical pathways are integrated into the whole treatment process through the proposed system. For the sake of seamless integration and physicians' preference, the interface of the system has been embedded in the existing physician workstation in three ways: (1) add the submenu of 'clinical pathway management' into the main menu of the workstation to enter the clinical pathway management system, (2) add 'patient pathway list' button into the patient information management function of the workstation, and (3) add 'current patient's pathway' button into the order entry function of the workstation.

The pathway information has been integrated into the treatment process and displayed through the interface for the physician. Figure 7 shows a diagram of the simplified clinical pathway of NSTACS. Each step in the pathway includes a detailed description with medical activity recommendations. Figure 8 shows the first step in clinical pathway of NSTACS for certain patients. The progress steps, various medical activities and related clinical knowledge for this disease have been defined in the knowledge repository. The system assists the physician with pathway information and suggests relevant medical behaviours for ticking and customising in the treatment process. In addition, the pathway status is updated constantly and displayed to the physician. In this way, the physician can be efficiently aware of the real-time pathway status and keep track of the treatment progress accordingly.

Figure 8 shows the patient-specific medical activities in the first step of NSTACS. It is obtained by combining of various forms of decision support including implicit (structured data entry) and explicit (plausibility checks, alerts and dash boards) reminders. The decision support provided by the system covers pathway processes, indices and medical activities in a clinical pathway, such as examination, evaluation, nutrition, observation, permitted activity, nursing, surgery and consultation, as well as the order entry and other clinical documentation related decision support. Medical activities corresponding to the pathway recommendation above are listed and marked with a checkbox for selecting and placing an order for execution directly. 


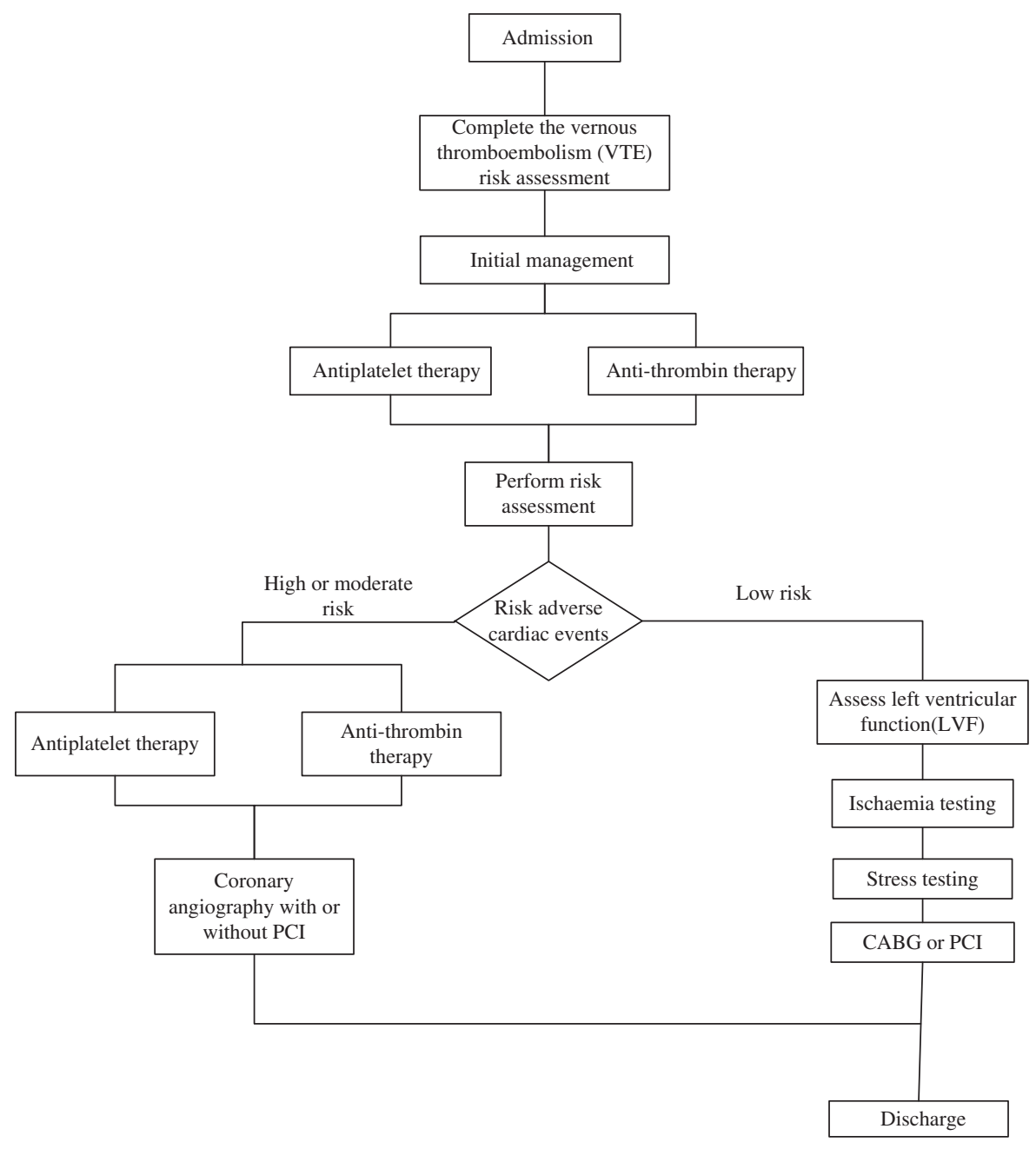

Figure 7 A simplified clinical pathway for NSTACS.

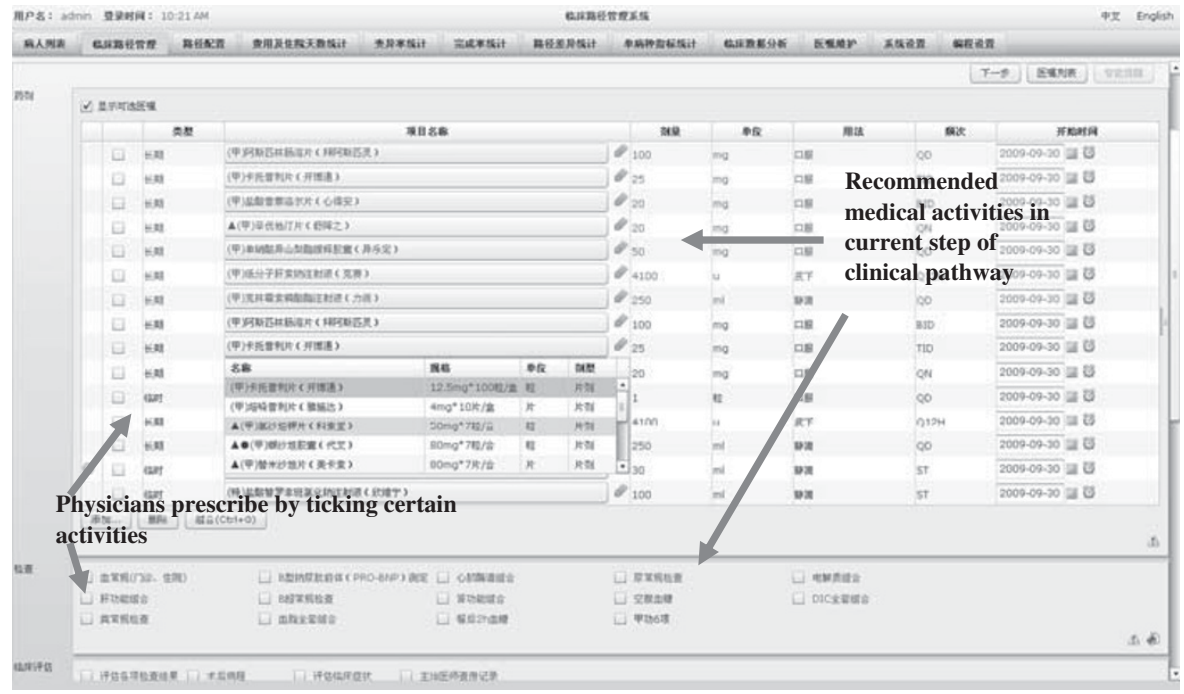

Figure 8 Interface for the integrated clinical pathway management. 
Through the interaction between pathway engine and information system, physicians can prescribe directly on this interface, which will further submit the prescription automatically to corresponding information systems. It is safer and more efficient than the traditional way where a printout of a prescribed order from the physician is executed by a nurse in a different department separately.

\section{Evaluation method}

Evaluation method design In order to implement and evaluate this approach, the integrated clinical pathway group consisting of physicians and nurses from the cardiology department, oncology department, orthopaedics department, gynaecology and obstetrics department has been set up. Staff from the IT department also joined the group to implement the system. The physicians are the main user of the system, who decide which activities should be executed in the treatment process based on the recommended pathway information. The physicians are delegated to take the overall decision and coordinate and delegate activities over the treatment process through the integrated clinical pathway system. The nurses are not the main user of the system, but they review the orders generated by the system and acknowledge their accomplishment.

The knowledge for clinical pathways for the six diseases is provided by the health professionals. The information department staff collaborate with health-care professionals in getting the pathway information into the knowledge base. The group meets every 2 weeks to communicate issues that have occurred during the implementation. When patients are admitted to hospital, they will be evaluated against the criteria for entering a pathway treatment. The information on the patients who are suitable for pathway treatment will be transferred into the integrated pathway system from electronic medical record.

In this prospective cohort study, a comparison was made between the medical quality in the treatment process before and after implementation of an integrated clinical pathway system. Data was collected before the implementation for a 6-month period from October 2009 to April 2010. There was a period of 1 month where there was no data collected in order to allow for the system implementation and training for related staff. A set of 6-month post-implementation data was collected from May to November 2010.

Main outcome measures Four lead physicians were designated to review all eligible pathway cases independently. The measures for medical quality were identified and further classified into categories based on ordering practice in HGSD and published literature (Potts et al, 2004). The main outcome measures include length of stay and medical errors. The length of stay indicates the average duration of the treatment process for a certain disease. Medical errors refer to any order that was incomplete, incorrect or inappropriate during
Table 2 The main outcome measures for medical error

\begin{tabular}{|c|c|}
\hline Medical error & \\
\hline Potential adver & events \\
\hline $\begin{array}{l}\text { Duplicate } \\
\text { therapy }\end{array}$ & $\begin{array}{l}\text { Same therapy prescribed twice or two or more } \\
\text { therapies from the same class with no evidence- } \\
\text { based pathway to prove benefit from both }\end{array}$ \\
\hline $\begin{array}{l}\text { Inappropriate } \\
\text { dose }\end{array}$ & $\begin{array}{l}\text { On the basis of a } 10 \% \text { difference in pathway and } \\
\text { published dosing guidelines }\end{array}$ \\
\hline $\begin{array}{l}\text { Inappropriate } \\
\text { interval }\end{array}$ & $\begin{array}{l}\text { On the basis of differences found from pathway and } \\
\text { published dosing guidelines }\end{array}$ \\
\hline $\begin{array}{l}\text { Inappropriate } \\
\text { route }\end{array}$ & $\begin{array}{l}\text { Therapy not available or not recommended to be } \\
\text { given in the route ordered }\end{array}$ \\
\hline Wrong drug & Incorrect drug ordered \\
\hline Wrong units & $\begin{array}{l}\text { Units are not correct for drug, diagnosis or dose } \\
\text { used (e.g., units } / \mathrm{kg} / \mathrm{min} v \mathrm{mcg} / \mathrm{kg} / \mathrm{min} \text { ) }\end{array}$ \\
\hline $\begin{array}{l}\text { Drug } \\
\text { interaction }\end{array}$ & $\begin{array}{l}\text { Documented drug interaction between two } \\
\text { medications that deems drug ineffective or } \\
\text { contraindicated (e.g., beta-blocker with beta- } \\
\text { agonist) }\end{array}$ \\
\hline Allergy & Documented allergy to drug ordered \\
\hline
\end{tabular}

Prescribing error

Missing

information

Missing route, interval, concentration, rate or dose that results in an incomplete order

Illegible Unable to read, required further interpretation

the treatment process. As shown in Table 2, errors were classified as potential adverse events and prescribing error. A potential adverse event is defined as any error that results in patient injury if it reaches the patient. These are errors where physicians provide incorrect or inappropriate information in the treatment process. This also includes instances in which the physician fails to account for patient-specific information (e.g., allergy). Prescribing errors are defined as errors in which inadequate information is provided or further interpretation (e.g., illegibility) is required for the order to be processed. The specific errors in each category are listed in Table 2.

All of the data above is collected from online web-based report forms completed by physicians monthly. It is mandatory for all the physicians to report any medical errors. The patient safety committee in the hospital will monitor the medical quality by inspecting the selected medical records regularly. If there are any unreported medical errors, the responsible physician will be warned by the committee. The report forms contain the following information: patient information, medical order information and medical error categorisation (medical activity class involved, categorisation of medical error and possible cause of medical error). Furthermore, in this pre-test and post-test study, the evaluation data is always collected through these online forms in order to keep the consistency for the process reporting medical errors with the implementation of the new clinical pathway system. 


\section{Results}

$\mathrm{A} \chi^{2}$ analysis and Fisher exact test for smaller sample sizes are used for data comparison before and after the implementation of the integrated clinical pathway system. A total of 342 pathway cases involving 342 patients were analysed throughout the study period. A total of 168 patients were evaluated during the pre-implementation period of the integrated clinical pathway system and 174 patients were evaluated during the post-implementation period. In the pre-implementation period, a total of 252 errors were identified. After additional classifications, 1.1 errors per pathway case were identified as potential adverse events, 0.6 errors per pathway case were identified as prescribing error. A total of 53 errors were identified during the post-implementation period; 0.1 errors per pathway case were identified as potential adverse events and 0.4 errors per pathway case were categorised as prescribing error. Overall, the integrated clinical pathway management system significantly reduced medical error, which results in a $70.9 \%(P<0.001)$ reduction in various errors in the treatment process. Furthermore, because of the reduction of medical errors, the average length of stay has been reduced. The mean length of stay is $12.8 \pm 5.2$ days for the pre-implementation group and $10.1 \pm 4.7$ days for the postimplementation group. The result details are shown in Table 3.

\section{Limitations}

The pre-post designs are commonly used to evaluate the effectiveness of clinical pathways. The simple pre-post study designs tend to overestimate intervention effects reported (Bero et al, 2009; Rotter et al, 2012). As clinical pathway is evidence-based and will be improved by health-care professionals continuously learning from deviations and practical treatment processes, the evolution of the pathways in this study will affect the outcome to some extent. In this case study, there are some updates for several pathways that have effects on medical quality improvement, for example, reducing pathway deviation and length of stay. In addition, that physicians become more familiar with pathway knowledge during the study is another minor factor leading to some reduction in medical errors.

\section{Related research}

This section gives a brief state of the art review of the area and compares the related researches with the work presented in this paper. Currently, IT has been recognised by many researchers as a useful tool for clinical decision support. They are used to improve medical performance and pathway compliance by delivering relevant advice in a timely manner (Johnston et al, 1994; Elson \& Connelly, 1995). These capabilities rely on the structured clinical data and rules that trigger appropriate recommendations (Zielstorff, 1998; Lenz \& Reichert, 2005).

Generally, most of the clinical decision supports are in the form of reminder systems and computerised physician order systems. Reminder systems are systems that can generate alerts and reminders in medical practice, such as monitoring the current status of a patient by generating alerts if some parameter, or a combination of parameters, is giving danger signals (Bates et al, 2001), or generating reminders to ensure that planned actions are not forgotten (McDonald, 1976). Computerised physician order entry (CPOE) systems allow clinicians to electronically enter medication orders. Friedlin et al (2007) established the CPOE with clinical decision support that is the production system including rules classified by G-Care rule types, which can also trigger alerts and reminders while placing medical orders. However, these decision supports can only support limited types of treatment activities rather than the whole treatment process. The computerisation of the clinical pathway became more important and some clinical pathway management systems have been designed

Table 3 Overall medical errors before and after the integrated clinical pathway management system

\begin{tabular}{|c|c|c|c|c|c|}
\hline & \multicolumn{2}{|c|}{ Pre-implementation $(n=168)$} & \multicolumn{2}{|c|}{ Post-implementation $(n=174)$} & \multirow[t]{2}{*}{$P$ Value } \\
\hline & Total number & $\begin{array}{c}\text { Number per } \\
\text { pathway case }\end{array}$ & Total number & $\begin{array}{l}\text { Number per } \\
\text { pathway case }\end{array}$ & \\
\hline Potential adverse events & 184 & 1.1 & 17 & 0.1 & $<0.001$ \\
\hline Duplicate therapy & 41 & 0.24 & 0 & 0 & $<0.001$ \\
\hline Inappropriate dose & 53 & 0.32 & 5 & 0.03 & $<0.001$ \\
\hline Inappropriate interval & 24 & 0.14 & 4 & 0.02 & 0.01 \\
\hline Inappropriate route & 6 & 0.03 & 0 & 0 & $<0.001$ \\
\hline Wrong drug & 6 & 0.03 & 0 & 0 & $<0.001$ \\
\hline Wrong units & 32 & 0.19 & 8 & 0.05 & $<0.001$ \\
\hline Drug interaction & 31 & 0.18 & 0 & 0 & $<0.001$ \\
\hline Allergy & 1 & 0.01 & 0 & 0 & 0.49 \\
\hline Prescribing error & 68 & 0.4 & 36 & 0.21 & $<0.005$ \\
\hline Missing information & 46 & 0.27 & 25 & 0.14 & 0.07 \\
\hline Illegible & 22 & 0.13 & 11 & 0.07 & 0.07 \\
\hline
\end{tabular}


(Chu, 2001; Mathe et al, 2009). However, current clinical pathway systems only computerise the pathway documents without detailed knowledge specification and sharing, thus lack of integration with existing IT systems and coordination among health-care staff. Therefore, the problems of clinical pathway implementation still exist and pathway still cannot be used to its full extent in improving medical quality.

Kuhn and colleagues (Kuhn et al, 2003; Lenz \& Kuhn, 2004; Blaser et al, 2007) have developed the adapted software engineering process to achieve an IT-supported pathway that is aligned to the clinical workflows, where patient-specific pathway information can be integrated into documentation with rule-based recommendation. However, the whole reengineering process is time consuming and it is impractical to redesign the whole system in the hospital. Therefore, a flexible approach is needed for comprehensive decision support in the treatment process. HL7 interoperability standard is thus adopted for the implementation of a clinical decision support system providing point-of-care chronic disease management at regional hospitals (Lobach et al, 2007). This system has the feature of portability and scalability that could facilitate integration throughout the regional health system and improve efficiency.

As discussed above, the current clinical decision systems mainly focus on the computerisation of clinical documents or the system reengineering process, but there is no step towards an integrated approach of incorporating clinical pathway knowledge with treatment process and the existing information systems. The introduction of the co-design of clinical pathway knowledge and the integrated pathway management system, as well as the use of agent technology to realise the patient personalised pathway support and the interaction among existing information systems for building a co-operating team, gives an added value to this integrated clinical pathway management approach and differentiates it from the other clinical decision systems in its effectiveness in improving medical quality.

\section{Discussion and conclusions}

Clinical pathways have been introduced in many health-care organisations around the world with the aim of improving medical quality. Integration of the clinical pathway knowledge with hospital information system and treatment process has proven successful in supporting medical practices, reducing medical errors and thereby improving medical quality. However, current implementation of clinical pathway is separated from existing information systems and treatment process. The challenges and the shortcomings of current health-care information systems are due to the lack of integration of adequate pathway knowledge with existing information systems. However, there is limited research on integrating the informal medical quality improvement factors, formal pathway knowledge and technical information systems together to improve health-care quality.

The innovation of this study is twofold: the co-design methodology for integrated clinical pathway management and the layered system architecture design applied in the pathway management area. The integrated system design is based on OS in which organisations are viewed as information systems. From this perspective, researchers have made efforts in sourcing a solution to treat IT systems and business process as one integral unit and hence minimising the gap between them. Liu et al (2002) proposes the concept of co-design of business and IT systems that require IT systems to meet the business requirements support the business process and adapt to changes in business practices. The hypothesis is that the organic integration of IT into the business processes will allow both systems to evolve naturally, which requires the codesign of the IT systems and business process. The paper by De Moor (2002) drawing input from OS and language action perspective discusses that an information system should be formed on the basis of a socio-technical system dependent on the professional community interrelated to an IT system. The RENISTS introduced in the paper enables the users to gain an understanding of the workflow and the support by IT, therefore to be able to specify the sociotechnical system. An approach of co-design of clinical pathway knowledge and the integrated pathway management derived from the above viewpoints is presented in this study. The design of the integrated system architecture and the proposition of specific methods to make the clinical pathway system highly supportive of treatment processes is the main innovation of this study.

Another innovation of this study is the layered integrated system architecture design in health-care systems. The layered architecture has been used extensively as it provides a model to create a flexible and reusable application (Fowler, 2002). In the health-care domain, Yang et al (2006) use multi-layer architecture to implement the outpatient hospital information systems for robustness and scalability. Otto et al (2005) use multi-layer architecture in a wireless sensor network system for ambulatory health status monitoring. In this study, because of the complex and dynamic nature of clinical pathway knowledge, the layered system that integrates IT systems into treatment process seamlessly has been designed. The layered system will not only present the technical systems, but also integrate the formal process and the informal culture factors. Furthermore, the design of the layered integrated pathway system, on the one hand, preserves the original system investment and, on the other hand, retains the scalability for a broader range of medical quality management.

The integrated clinical pathway system in this study significantly reduced all categories of errors and length of stay, which shows that medical quality can be effectively improved through this approach. Compared with other existing approaches of clinical decision support systems, such as reminder system and computerised physician 
order entry system (McDonald, 1976; Johnston et al, 1994; Elson \& Connelly, 1995; Bates et al, 2001), this approach and result has demonstrated a better pathway knowledge support in the whole treatment process as well as better improvement in medical error and length of stay. Moreover, this study also contributes to the design approach of the socio-technical system by integrating the informal, formal and technical levels in the organisation.

On the other hand, this research faces some common issues for the socio-technical system. The integration of medical knowledge into treatment process not only lies in the integrated system, but also depends on the informal level of the organisation. As discussed in the sections 'Motivation of co-design methodology - The complexity of treatment processes' and 'Architectural design', the patient safety culture and the practical treatment process constitute the informal level of integrated clinical pathway management. Patient safety culture is an important issue in health care and much research has been undertaken for promoting the culture and improving medical quality (Fleming \& Wentzell, 2008; Ginsburg et al, 2009). For example, the National Patient Safety Agency has identified seven key steps to promote patient safety culture (NPSA, 2004). In this research, a framework in the informal level on how to establish the shared belief and value for high medical quality and patient safety in clinical pathway management will be needed in future work.

Furthermore, although the integrated clinical pathway system can combine the formal pathway knowledge with IT systems effectively, there are still existing informal issues on knowledge management in the treatment process. These informal issues involve culture misfit, educational

\section{About the Authors}

Weizi Li is Research Fellow in the Informatics Research Centre, School of Business Informatics, Systems and Accounting, within Henley Business School at University of Reading. She holds a Ph.D. degree in health-care informatics and information systems. Her research focuses on organisational semiotics (OS), systems integration, digital hospital and knowledge management. She has published papers actively in health care informatics in academic journals and conferences. She has been participating in a number of research projects in information integration, systems engineering and integration, hospital information systems, and process modelling and optimisation.

Kecheng Liu is a Fellow of the British Computer Society and Professor of Applied Informatics. He is the Director of the Informatics Research Centre, and the Head of School of Business Informatics, Systems and Accounting, within Henley Business School at University of Reading, U.K. He has published over 180 papers in journals and conferences, and been serving in a number of editorial boards of journals. He is one of the key figures in OS. His research includes information systems, pervasive informatics and intelligent systems. backgrounds and hierarchical thinking(Mettler \& Rohner, 2009), which can have a strong effect meaning that healthcare professionals might not follow the suggested pathway provided by the integrated system. These informal issues have an important impact on socio-technical systems. In this study, the health-care professionals need to note the reasons for not following the suggested pathway and all the data for any deviation and reasons will be collected and analysed regularly. The analysis results will further be used to update the formal medical knowledge and be integrated into information systems.

The main users of the integrated clinical pathway management system are physicians, who prescribe according to the recommended pathway information during the treatment process. The execution of the clinical pathway is guided and coordinated by the system. However, the procedures of preparation or dispensing that are performed by nurses cannot be supported by this system. Therefore, further research work is needed to reduce the errors in preparation and dispensing during the execution of clinical pathway. As clinical pathway is evidence-based combining the treatment process steps with long-term informal medical experiences, future work would involve how the pathway knowledge in the integrated system could be further refined and improved by continuously monitoring, analysing, mining and learning from the deviations. This plays an important role for the sociotechnical system solving informal issues in treatment processes. The clinical pathway will be integrated into a broad range of systems providing a wider range of support (e.g., care at home and mobile hospital), which will further improve medical quality.

Hongqiao Yang is the Director of the Information Centre in one of the largest hospitals in China. He conducts the postdoctoral research in computer science and technology in China. He is a also Visiting Scholar in the Informatics Research Centre, University of Reading for postdoctoral research. He has been involved in co-supervising Ph.D. projects in the Informatics Research Centre, University of Reading. His research interests fall in the field of artificial intelligence, information system design, complex systems modelling and simulation, and OS. He has been in charge of many national and ministerial research projects and has been working on the development of hospital information technology for past years.

Changrui Yu is an Associate Professor in School of Information Management and Engineering at Shanghai University of Finance and Economics. He is also a Research Fellow in Informatics Research Centre, University of Reading. His current research interests include management information system, software engineering and ontology. He has published over 30 papers in the journals and conferences. 


\section{References}

ABIDI SSR (2008) Healthcare knowledge management: the art of the possible. In Proceedings of the Conference on Knowledge Management for Health Care Procedures (RIAÑO D Ed) pp 1-20, Springer, Berlin, Heidelberg, Germany.

BAACKE L, METTler T and ROHNER P (2009) Component-Based Process Modelling in Health Care, ECIS 2009, Proceedings: 38, Verona, Italy.

BARJIS J, GUPTA A and SHARDA R (2010) Knowledge work and communication challenges in networked enterprises. Information Systems Frontiers 13(5), 615-619.

Bates DW, Cohen M, Leape LL, OVerhage JM, Shabot MM and Sheridan T (2001) Reducing the frequency of errors in medicine using information technology. Journal of the American Medical Informatics Association 8(4), 299-308.

BERO L et al (2009) Cochrane Effective Practice and Organisation of Care Group (Cochrane Group Module). About The Cochrane Collaboration (Cochrane Review Groups (CRGs)). The Cochrane Library, John Wiley, Oxford.

BLASER R et al (2007) Improving pathway compliance and clinician performance by using information technology. International Journal of Medical Informatics 76, 151-156.

CHU S (2001) Computerised clinical pathway as process quality improvement tool. Studies in Health Technology and Informatics $\mathbf{8 4}$ (Pt2), 1135-1139.

COFFEY R, RiChaRdS I, REMMERT C, LEROY S, SCHOVILLE R and BALDWIN P (1992) An introduction to critical paths. Quality Management in Health Care 14(1), 45-55

CROMBIE I and DAVIES H (1993) Missing link in the audit cycle. Quality in Health Care 2(1), 47-48.

DARDEN L (2002) Strategies for discovering mechanisms: schema instantiation, modular subassembly, forward/backward chaining. Philosophy of Science 69(3), S354-S365.

DE MOOR A (2002) Language/action meets organisational semiotics: situating conversations with norms. Information Systems Frontiers 4(3), 257-272.

ELSON R and CONNELLY D (1995) Computerized decision support systems in primary care. Primary Care 22(2), 365-384.

FIPA. (2002) FIPA abstract architecture specification. [WWW document] http://www.fipa.org/specs/fipa00001/index.html (accessed 15 April 2013).

FLEMING M and WeNTZELL N (2008) Patient safety culture improvement tool: development and guidelines for use. Healthcare Quarterly 11(Sp), 10-15.

FOWLER M (2002) Patterns of Enterprise Application Architecture. AddisonWesley Longman, Boston, MA.

Friedin J, Dexter PR and Overhage JM (2007) Details of a successful clinical decision support system. AMIA Annual Symposium, pp 254-258, American Medical Informatics Association.

Ginsburg L, Gilin D, TregunNo D, Norton PG, Flemons W and Fleming M (2009) Advancing measurement of patient safety culture. Health Services Research 44(1), 205-223.

HL7. (2007) Health level seven international. [WWW document] http:// www.hl7.org/ (accessed 27 Febuary 2012).

HURLEY KF and ABIDI SSR (2007) Ontology engineering to model clinical pathways: towards the computerization and execution of clinical pathways. CBMS '07: Proceedings of the Twentieth IEEE International Symposium on Computer-Based Medical Systems. Maribor, Slovenia, IEEE Computer Society, Washington, DC.

ISERN D, SÁNCHEZ D and MORENO A (2010) Agents applied in health care: a review. International Journal of Medical Informatics 79(3), 145-166.

JOHNSTON ME, LANGTON KB, HAYNES RB and MATHIEU A (1994) Effects of computer-based clinical decision support systems on clinician performance and patient outcome: a critical appraisal of research. Annals of Internal Medicine 120(2), 135-142.

KITCHINER D and BUNDRED P (1996) Integrated care pathways. Archives of Disease in Childhood 75(2), 166-168.

KIZER KW (1999) Large system change and a culture of safety. In Enhancing Patient Safety and Reducing Errors in Health Care. National Patient Safety Foundation, Chicago, IL.

KUHN KA, LENZ R, ELSTNER T, SIEGELE H and MOLL R (2003) Experiences with a generator tool for building clinical application modules. Methods Information in Medicine 42(1), 37-44.
LENZ R and KUHN KA (2004) Towards a continuous evolution and adaptation of information systems in healthcare. International Journal of Medical Informatics 73(1), 75-89.

LENZ R and REICHERT M (2005) IT support for healthcare processes. Business Process Management. Lecture Notes in Computer Science 3649, 354-363.

LI W, LIU K, LI S and YANG H (2008) Normative modeling for personalized clinical pathway using organizational semiotics methods. International Symposium on Computer Science and Computational Technology. Vol. 2, pp 3-7. IEEE Computer Society, Shanghai, China.

LI W, LIU K, LI S and YANG H (2009) An agent based approach for customized clinical pathway. International Forum on Information Technology and Applications. Vol. 2, pp 468-472. IEEE Computer Society, Chengdu, China.

LI W, LIU K, LI S and YANG H (2010) A semiotic multi-agent modeling approach for clinical pathway management. Journal of Computers 5(2), 266-273.

LIU K (2000) Semiotics in Information Systems Engineering. Cambridge University Press, Cambridge.

LIU K, SUN L and BENNETT K (2002) Co-design of business and IT systems - introduction by guest editors. Information Systems Frontiers 4(3), 251-256.

LobaCh D, KaWAMOto K, ANSTROM K, Russell M, WoOdS P and SMith D (2007) Development, deployment and usability of a point-of-care decision support system for chronic disease management using the recently-approved $\mathrm{HL7}$ decision support service standard. Studies in Health Technology and Informatics 129(Pt2), 861-865.

MATHE JL et al (2009) A model-integrated, guideline-driven, clinical decision-support system. IEEE Software 26(4), 54-61.

MCDONALD CJ (1976) Protocol-based computer reminders, the quality of care and the non-perfectability of man. The New England Journal of Medicine 295(24), 1351-1355.

METTLER T and ROHNER P (2009) An analysis of the factors influencing networkability in the health-care sector. Health Services Management Research 22(4), 163-169.

MUSCHOLL M (2005) Integrated clinical pathways in health information systems - an architectural concept. Professional Knowledge Management, Lecture Notes in Computer Science. Springer 3782, 349-359.

NEALON J and MORENO A (2003) Agent-based applications in health care. In Applications of Software Agent Technology in the Health Care Domain. Birkhäuser Verlag, Basel, Switzerland, pp 3-18.

NPSA: National Patient Safety Agency. (2004) Seven steps to patient safety: the full reference guide Nationa. Second print, August.

Otto C, Milenkovic A, SANDERS C and Jovanov E (2005) System architecture of a wireless body area sensor network for ubiquitous health monitoring. Journal of Mobile Multimedia 1(4), 307-326.

Potts AL, BARR FE, GreGory DF, WriGht L and PATEL NR (2004) Computerized physician order entry and medication errors in a pediatric critical care unit. Pediatrics 113(1Pt1), 59-63.

Rotter T, Kinsman L, James E, Machotta A and Steyerberg E (2012) The quality of the evidence base for clinical pathway effectiveness: room for improvement in the design of evaluation trials. BMC Medical Research Methodology 12, 80, doi:10.1186/1471-2288-12-80.

STAMPER RK (2001) Organisational semiotics: informatics without the computer? In Information, Organisation and Technology: Studies in Organisational Semiotics (LIU K, CLARKE RJ, ANDERSEN PB and STAMPER RK, Eds) pp 115-171, Kluwer Academic Publishers, Boston, MA.

Stronks A and GALVEZ E (2007) Hospitals, language, and culture: A snapshot of the nation. Exploring Cultural and Linguistic Services in the Nation's Hospitals: A Report of Findings. Sponsors: The California Endowment; the Joint Commission, United States.

VANHAECHT K (2007) The impact of clinical pathways on the organisation of care processes. PhD thesis, Katholieke Universiteit Leuven, Flanders, Belgium.

VEGODA P (2002) Introducing the IHE (integrating the healthcare enterprise) concept. Journal of Healthcare Information Management 16(1), 22-24.

Vincent C, Neale G and Woloshynowych M (2001) Adverse events in British hospitals: preliminary retrospective record review. $B M$ 322(7285), 517-519. 
YANG H and LI W (2009a) Modeling requirement driven architecture of adaptive healthcare system based on semiotics. International Forum on Information Technology and Applications. Vol. 2, pp 723-727. IEEE Computer Society, Chengdu, China.

YANG H and LI W (2009b) An ontology-based approach for data integration in regionally interoperable healthcare systems. The 11 th International Conference on Informatics and Semiotics in Organisations. INSTICC publisher, Beijing, China, Portugal.

YANG H, LIU K and LI W (2010) Adaptive requirement-driven architecture for integrated healthcare systems. Journal of Computers 5(2), 186-193.

YANG TH et al (2006) A scalable multi-tier architecture for the National Taiwan University Hospital Information System based on HL7 standard. 19th IEEE International Symposium on Computer-Based Medical Systems. Salt Lake City, UT, IEEE Computer Society.

ZIELSTORFF RD (1998) Online practice guidelines: issues, obstacles, and future prospects. Journal of the American Medical Informatics Association 5(3), 227-236

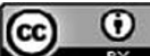

This work is licensed under a Creative Commons Attribution 3.0 Unported License. To view a copy of this license, visit http://creativecommons. org/licenses/by/3.0/ 